\section{Operational Efficiency of Urban Malaria Scheme in a District of Central Gujarat, India: An Evaluation Study}

\section{Abstract}

Background: Improving the efficiency of the Malaria control program can help combat malaria in urban areas, which still remains a major public health problem in India, affecting all spheres of human life.

Methodology: Assessment of the overall functioning of Urban Malaria Scheme run by Municipal Corporation (MC) in a district of Central Gujarat, India, was carried out over 8 months(November 2011 to June 2012) using a semi-structured instrument by observation, inspection, record review of blood smear examination and target examination rates and interviews of laboratory technicians (LT) and pharmacists. Two slide-positive individuals from each clinic and one community leader from each ward was interviewed regarding the functioning of malaria clinics.

Results: Diagnosis and treatment facilities for malaria provided in 13 corporation dispensaries were observed. Though manpower was adequate, there was lack of active surveillance in the urban areas. The basic infrastructure facilities were inadequate in half of the dispensaries and facilities for Blood Slide Collection (BSC) and staining were found to be very poor. Majority of the dispensaries did not have any idea about the blood examination rate targets and the new treatment guidelines for malaria had not reached the clinics till the time of visits. Radical treatment (RT) was found to be inadequate and unsupervised. Half of the community leaders were unaware about the availability of diagnostic facility for malaria at these dispensaries and had inadequate information about availability RT.

Recommendations: The vacant posts of LT need to be filled up at the earliest; the laboratory dispensaries to be equipped with adequate space, basic infrastructure facilities, adequate logistics and supplies for Blood Slide Examination (BSE) need to be looked into. Attention needs to be paid to follow the active surveillance activities in cities, following monthly targets distributing new malaria treatment guidelines and ensuring adequate and supervised RT.

Keywords: Malaria control; Operational efficiency; Urban; Urban malaria scheme Abbreviations: UMS: Urban malaria scheme; MC: Municipal corporation; BSC: Blood slide collection; RT: Radical treatment; LT: Laboratory technician; PS for MP: Peripheral Smear for Malarial Parasite; MO: Medical Officer; LMO: Lady Medical Officer; MBER: Monthly Blood Examination Rate; Pf: Plasmodium falciparum; Pv: Plasmodium vivax.
Shobha Misra, Kalpita

Shringarpure, Parag Chavda

and Dipak Solanki

Baroda Medical College, Vadodara, Gujarat, India

Corresponding author: Shobha Misra

झ drshobhamisra@gmail.com

Baroda Medical College, Vadodara, Gujarat, India.

Tel: +919998787375

Citation: Misra S, Shringarpure K, Chavda $P$, et al. Operational Efficiency of Urban Malaria Scheme in a District of Central Gujarat, India: An Evaluation Study. J Infec Dis Treat. 2016, 2:2.

\section{Introduction}

The history of malaria elimination dates back to the late nineteenth century with the discovery of Plasmodium parasite and transmission of malaria through mosquito vectors [1]. Even today, malaria does not cease to be a major public health problem 
in India. 75 million cases and 0.8 million deaths werereported from India before the launch of National Malaria Control Program in 1953 [2-4]. India contributes $70 \%$ of malaria cases and $69 \%$ of malaria deaths in the South-East Asia Region [5]. However, there has been a scale up in malaria control activities due to increased interest in research activities to find newer strategies for malaria control [1]. Thus, in the last 10 years, total malaria cases have reduced by 42\%, (1.92 million in 2004 to 1.1 million in 2014) and deaths have reduced by $40.8 \%$ (from 949 to 562) [5].

One of the goals of the National Framework for Malaria Elimination in India during 2016-2030 is to eliminate malaria throughout the country by 2030 (Zero indigenous cases) [5]. The National Vector Born Disease Control Program strives to control malaria through early detection and prompt and adequate treatment, vector control measures and behavioral change communication [4].

Although there has been a rapid decline in the morbidity and mortality associated with malaria, one of the major challenges is urbanization that leads to a rise in malaria in urban areas $[2,3,5]$. South-Asia is at a risk of urban and peri-urban malaria, with nearly one hundred thousand cases of malaria being reported every year in these areas. Gujarat is one of the states with population in a few districts at high risk for malaria [5].

Though the health infrastructure is well developed in per-urban areas, malaria situation is influenced by poor sanitary condition and low socio-economic groups living in unplanned settlements and hence prone to epidemics periodically $[2,3,5]$.

Control of malaria was thought to be an important strategy in urban areas, complementary to the National Malaria Eradication Program (NMEP) for rural areas. Modified Plan of Operation (MPO) was designed and submitted to the Cabinet to tackle the malaria situation in both urban and rural areas in the country simultaneously. The proposal to control malaria in towns was named as Urban Malaria Scheme which was approved during 1971. The Urban Malaria Scheme is a centrally sponsored program being implemented mainly by local administrative authorities/ Municipalities under the active supervision of state health authorities [4].

A proper functioning and roll out of this program can keep malaria under control [6]. The whole edifice of malaria control is based on accurate laboratory diagnosis of blood smear form fever cases. The bottlenecks in malaria control mainly relate to the time lag at various stages commencing from collection of blood smear from fever cases to the administration of radical treatment to the positive cases.

Toourknowledge, there are hardly anyoperational research studies conducted hitherto globally or in India that comprehensively cover the entire malaria control program in urban areas [1,4,6-8]. So, a study to evaluate the Operation Efficiency of Urban Malaria Scheme (UMS) under Municipal Corporation (MC) of a district in Central Gujarat was carried out to assess the basic facilities available at Malaria clinic including the condition of microscopes, assessment of efficiency and skill of lab technicians and finding out the time lag between collection and examination of blood smears from the records maintained at Malaria clinics.

\section{Methodology}

Malaria control activities can be broadly classified into two components-Vector Control and Early Diagnosis and Prompt Treatment (EDPT) for reduction in morbidity and mortality due to malaria. The detailed qualitative part of the study on vector control activities has been described elsewhere [3].

In order to get an in-depth understanding of the overall functioning of UMS run by MC, a cross-sectional study using a semi-structured instrument was used. The study was carried out over a period of 8 months from November 2011 to June 2012. In this city, diagnosis and treatment facility for malaria is available through a network of Corporation, Non-Governmental Organizations (NGOs) and Private clinics. There are a total of 13 corporation dispensaries spread across 12 wards of the city that are equipped to provide diagnostic and treatment facility for malaria. The quantitative part of the study was conducted in these 13 dispensaries with prior permissions from the Chief Medical Officer and the district for better facilitation.

For assessment of EDPT component, information was collected by visiting these malaria clinics run by the Municipal Corporation. The assessment tools used during the visits were; observation, inspection, review of records to compare the targets and achievements of monthly blood examination rates, reviewing records of blood smear examinations, interviewing the Laboratory Technician (LT) and Pharmacists and observing the administration of radical treatment (RT) by staff at malaria clinic.

The individuals to be interviewed were selected at random from the records of last one month from the date of visit to minimize recall bias. Consent for participation in this study was obtained from the patients after explaining the purpose of the interview and the study.

Assessment of functioning of Malaria Clinic was carried out based on the following parameters.

- Basic facilities were considered adequate where the 5 observatory findings were met viz. availability of separate room for laboratory, availability of working table with adequate space, availability of revolving chair/ stool, facilities for keeping laboratory records and other materials and Sufficient natural light (from north side) or artificial light

- Microscope-Availability of functional microscope at the clinic

- Laboratory records-Availability of all 5 malaria forms (MF) and 4 malaria registers

- Blood smear collection (BSC) and staining materialsRegular supply of stains, availability of filter, availability of other materials like glass slides, pricking needles and spirit.

- Staff-Availability of one full-time laboratory technician during the entire previous year.

The functioning of LT was assessed for their role in UMS on the basis of parameters like training received, ability of preparing good quality peripheral smear for Malarial Parasite (PSMP), satisfactory maintenance of microscope and materials required for PSMP and malaria specific registers being maintained on daily basis. 
Any microscope fulfilling the following two criteria; was labeled as being maintained satisfactorily and any LT maintaining the microscope satisfactorily was regarded as having good efficiency; oil immersion lens and eye-piece free from dust and opacity and coarse and fine adjustment in good working condition.

Any LT using freshly prepared stain (prepared on daily basis) and making use of filters for staining PS for MP and keeping all materials needed for preparing PS for MP neat and clean was regarded as satisfactorily maintained materials.

Good quality peripheral smear for malarial parasite was assessed based on the following criteria: presence of both thick and thin smear on slide; serial number of slide clearly legible on thin smear; thick smear of about $1 \mathrm{~cm}$ in diameter and having light blue stain and violet iridescence; thin single layered and tongue shaped smear; and clean glass slides (old and new) free from dust, dirt, greasy spots and scratches. Any LT preparing good quality PS for MP was considered as having good skill for PSMP preparation.

Time lag form BSC to administration of Presumptive treatment in case of slide negative individuals and form BSC to administration of Radical Treatment in case of slide positive individuals was recorded from malaria registers and was verified for correctness by talking to the slide positive and slide negative individuals and discrepancy if any, was also noted down. Time lag at different stages from BSC to administration of RT in case of slide positive individuals was noted down from dispensary records.

The Operational Efficiency of UMS in respective wards was evaluated by interviewing community leader on parameters like availability of diagnostic and treatment facility for malaria, availability of active surveillance activities.

\section{Data entry and analysis}

Quantitative data collected by the above methods was entered into MS Excel 2003-2007 and the analysis in terms of descriptive statistics; percentages, proportions and scores was done using Epilnfo(TM) 3.5.4 [9].

\section{Limitations of the study}

Recall bias may have occurred during the interviews of patients, however, this was minimized by keeping the period between the occurrence and interview short. Survey was carried out during non-transmission season where few cases were reported. People also avail diagnostic and treatment facilities for malaria from private laboratories and clinics including NGO run clinics, data from which is neither sent to nor collected by the corporation authorities.

\section{Results and Discussion}

There are 13 corporation dispensaries spread across 12 wards of the city that are equipped to provide diagnostic and treatment facility for malaria, which were included in this study. The oldest dispensary was functional since 1951, while the most recent since 2004. Of these, 8 were functional full time, while the rest of them were part time, catering to a population in the range of 10,000 to $4,50,000$. The findings from visits to the corporation dispensaries which focus mainly on the diagnostic and treatment facility for malaria are described in this article.

\section{Malaria clinics}

All the visited dispensaries had at least one full time Medical Officer (MO). In total there were $17 \mathrm{MOs}$ out of which, six were Lady Medical Officers (LMOs). Pharmacist's post was vacant in one of the dispensaries; LT posts were vacant at three clinics. Male Health workers and Supervisors were available at only three dispensaries. This situation was similar to the one observed in the dispensaries of rural areas of the district [6].

Total 12 pharmacists were interviewed for the study. Most of the pharmacists (10/12) were posted on regular basis. Less than half (5/12) of pharmacists interviewed had correct knowledge about the treatment of malaria. Ten LTs were interviewed, of which eight were posted on a regular basis. Of these LTs, seven had received training but none of them had received it since last two years.

Poor functioning of the clinics can be due to these understaffed clinics and inadequately trained staff for the Control of Vector Borne Diseases, which has proved to be an impeding factor in malaria control [10].

As shown in Table 1, majority (11/13) of the dispensaries had a functional microscope. Also majority of them (10/13) had maintained all the malaria specific registers. Basic facilities at the Malaria clinics were inadequate in almost half of the dispensaries (7/13). It was also observed that adequate space was lacking at majority of them as the laboratory was running in the same room which was also used for other activities. At most of the dispensaries, no separate room was available for laboratory. Despite these problems, at majority of the places the preparation and staining of blood smear (BS) and maintenance of records was found to be satisfactory.

\section{Malaria surveillance}

Barring two of the 13 dispensaries visited, rest of them had no idea of the target of monthly blood examination rates (MBER) to be achieved in case of outpatient (OPD) fever cases (Table 2). It was observed that almost all the fever cases coming to the clinics were subjected to blood smear examination for malaria diagnosis. As per the National vector borne disease control program (NVBDCP), in rural areas, $15 \%$ of OPD cases are fever cases, which should be tested for malaria [11]. This protocol can be followed in urban areas also. However, there is no such documented information. Two of the dispensaries were non-functional, the patients were sent to the nearby dispensaries. One of the dispensaries sent slides to the nearby dispensary for microscopic examination due to lack of LT. This shows the additional burden to the LTs during the transmission season, which may affect the efficiency of work.

Table 1 Assessment of functioning of laboratories at the corporation dispensaries visited.

\begin{tabular}{|c|c|c|}
\hline S No & Parameter & $\begin{array}{c}\text { No. of } \\
\text { Clinics }\end{array}$ \\
\hline 1 & Total clinics visited & 13 \\
\hline 2 & Clinics having adequate basic facilities for & 6 \\
\hline 3 & laboratory & 11 \\
\hline 4 & Clinics having working microscope & 10 \\
\hline 5 & Clinics employing correct technique for preparing & 10 \\
\hline
\end{tabular}


Table 2 Comparison of targets and achievements of Monthly Blood Examination Rate of the dispensaries visited in the district.

\begin{tabular}{|c|c|c|c|c|c|c|c|c|c|}
\hline \multirow{2}{*}{ Name of dispensary } & \multicolumn{3}{|c|}{ Active surveillance } & \multicolumn{3}{|c|}{ Passive surveillance } & \multicolumn{3}{|c|}{ Total } \\
\hline & Target & Achieved & $\%$ & Target & Achieved & $\%$ & Target & Achieved & $\%$ \\
\hline Fatehpura & $-*$ & 399.67 & - & - & 314.3 & - & - & 713.97 & - \\
\hline Wadi & 1326 & 508.8 & 38.9 & - & 19.3 & - & 1326 & 528.1 & 39.83 \\
\hline Jubelibaugh & - & 390.67 & - & - & 102.8 & - & - & 493.47 & - \\
\hline Manjalpur & - & 667.1 & - & - & 70.7 & - & - & 737.8 & - \\
\hline Navidharti & 1022 & 449.83 & 44 & 681 & 87.67 & 12.87 & 1703 & 537.5 & 31.56 \\
\hline Navayard & - & 493 & - & - & 275.3 & - & - & 768.3 & - \\
\hline Navapura & - & - & - & - & - & - & - & - & - \\
\hline Tandalja & - & 408 & - & 89.5 & 58.6 & 56.2 & - & 466.6 & - \\
\hline Akota & - & 335 & - & 45.6 & 28.5 & 40.1 & - & 363.5 & - \\
\hline Bawchawad & - & 397.66 & - & 139 & 95.83 & 38.6 & - & 493.43 & - \\
\hline Maneja & - & - & - & - & - & - & - & - & - \\
\hline Tarsali & - & 43 & - & - & 43 & - & - & 86 & - \\
\hline Makarpura & - & - & - & - & - & - & - & - & - \\
\hline
\end{tabular}

*'-` : No target set

No targets were fixed for active surveillance in most of the dispensaries (10/13). It was noted from the functionaries interviewed, that it was not feasible to carry out active surveillance in urban areas due to lack of manpower.

\section{Malaria treatment}

Medical Officers (MOs) at the dispensaries informed that copy of new guidelines for treatment of Malaria was not received by them, thus they were following the older guidelines of RT. At the clinic primary responsibility of administration of RT in the field lies with a field worker. The actual administration of RT in the field was irrational in terms of observance of even the old guidelines let alone the new one. This could be attributed partly to lack of staff and lack of training amongst the field workers [3].

\section{Response of slide-positive individuals about the illness and radical treatment}

As shown in Table 3, a total of 9 slide-positive individuals were interviewed, including 7 males and 2 females. Out of these 9 individuals, 3 had Plasmodium falciparum (Pf) and 6 had Plasmodium vivax (Pv) infection. Mean number of days between blood smear collection and administration of RT was 1.23 days. All the 9 individuals interviewed, had received RT. However, it was adequate only in one third and supervised in $11 \%$ of the cases.

\section{Time lag}

At the laboratories, the majority of the slides were received through passive surveillance. Time lag between BSC and examination was minimal as the slides were being examined on the same day. Whereas the mean time lag between blood slide examination and administration of RT was 1 day. Thus, at majority of the dispensaries, the RT was being administered within one day.

\section{Information from Pf positive cases}

The study period being non-transmission season for malaria, only $3 \mathrm{Pf}$ cases were reported during the period of study from the visited dispensaries. Out of these, only 2 could be traced at their home. On enquiry, it was found that neither the blood slides were collected from the family members nor there was follow up smear from patients. No survey activities were carried out by the corporation authorities on confirmation of these Pf positive cases in their area.

\section{Responses of the interviewed slide negative individuals about their illness}

Gender-wise responses of slide negative individuals interviewed, to the various inquiries with reference to their illness within past one month of the visit by investigator were reported. A total of 21 individuals were contacted, out of which 11 were males and 10 were females. All 21 individuals interviewed, responded in affirmation when inquired whether their blood was collected within the past month. All excepting 2 gave history of fever on same day or within last 15 days of blood collection. Mean time interval between onset of fever and blood smear collection (BSC) was 3.64 days, being 2.5 days in males and 4.9 days in females. Time lag was more in case of females.

It is imperative to note here that majority of the individuals interviewed contacted the dispensary on their own (passive surveillance) as active surveillance in urban areas is an extremely limited activity. So the time lag noted reflects patients own delay in seeking health care. Lack of supervised drugs was also one of the major issues observed in this study. Malaria control can be achieved not only through affordable access to malaria diagnosis and treatment, but also need improvement in laboratory tools, the health-seeking behavior and awareness of patient and more importantly, strengthening the diagnostic and treatment facility [1]. Case studies from Philippines have shown active surveillance to improve the operational efficiency of the program [8]. Importance of data for decision making and importance of active surveillance has been stressed in the Global Technical Strategy for Malaria [12]. All these aspects were seen lacking in the urban malaria dispensaries.

Awareness of community leaders regarding availability of facilities for malaria at dispensaries

Total 13 community leaders were interviewed, one from each ward. Questions pertained to the diagnosis and treatment 
Table 3 Responses of slide-positive individuals about the illness and radical treatment.

\begin{tabular}{|c|c|c|c|c|c|c|c|c|}
\hline \multirow[b]{2}{*}{ Gender } & \multirow[b]{2}{*}{ Age group } & \multirow{2}{*}{$\begin{array}{l}\text { Number of } \\
\text { individuals } \\
\text { interviewed }\end{array}$} & \multicolumn{2}{|c|}{ Species } & \multirow{2}{*}{$\begin{array}{l}\text { Mean no. of } \\
\text { days between } \\
\text { BSC and RT }\end{array}$} & \multirow[b]{2}{*}{ RT received } & \multirow[b]{2}{*}{ Adequate RT } & \multirow[b]{2}{*}{ RT supervised } \\
\hline & & & Pv & Pf & & & & \\
\hline \multirow{3}{*}{ Male } & Adult & 7 & 4 & 3 & 1.3 & 7 & 3 & 1 \\
\hline & Children & 0 & 0 & 0 & 0 & 0 & 0 & 0 \\
\hline & Total & 7 & 4 & 3 & 1.3 & 7 & 3 & 1 \\
\hline \multirow{3}{*}{ Female } & Adult & 1 & 1 & 0 & 1 & 1 & 0 & 0 \\
\hline & Children & 1 & 1 & 0 & 0 & 1 & 0 & 0 \\
\hline & Total & 2 & 2 & 0 & 1 & 2 & 0 & 0 \\
\hline \multirow{3}{*}{ Total } & Adult & 8 & 5 & 3 & 1.26 & 8 & 3 & 1 \\
\hline & Children & 1 & 1 & 0 & 0 & 1 & 0 & 0 \\
\hline & Total & 9 & 6 & 3 & 1.23 & 9 & 3 & 1 \\
\hline
\end{tabular}

BSC-Blood smear collection; RT-Radical treatment; Pv-Plasmodium vivax; Pf-Plasmodium falciparum

of malaria at the dispensaries in respective wards. Although majority of them (11/13) were aware about the corporation dispensary, more than half of them (6/11) were unaware about the availability of diagnostic facility for malaria at the dispensaries. Majority believed the time taken by dispensaries for diagnosis of malaria to be more than one day (4/5). It is also noted that almost two thirds of them were aware about the availability of RT (9/13).

\section{Perception of community leaders about active surveillance}

Only 6 out of 13 said that active surveillance was going on in their area. Five of them affirmed that the workers do inquire about fever cases. Four were aware about blood slides collection and 3 about tablets given after BSC.

\section{Perception of community leaders about vector control activities carried out in their area}

Various anti-adult, anti-larval and source reduction activities were inquired about. Around 25\% (3/13 and 4/13) community leaders were aware about anti-adult measures like fogging and spraying respectively. With reference to the time period since any of these two activities carried out last, about $25 \%(4 / 13)$ of the community leaders said that this time period was more than 6 months and an equal percentage said that it was less than 6 months. Knowledge of community leaders as a reflection of the community awareness seems to be poor. Adequate educational programs need to be initiated so as to achieve success of malaria control program [7].

\section{Conclusion and Recommendations}

To conclude, diagnosis and treatment facilities for malaria provided in 13 corporation dispensaries were observed. At least one Medical Officer was available at all dispensaries. Though manpower was adequate, there was lack of active surveillance in the urban areas. While working microscope and maintenance of registers were found to be satisfactory in majority of the centers, facility for BSC and staining were found to be very poor. Majority of the dispensaries did not have any idea about the blood examination rate targets and the new treatment guidelines for malaria had not reached the clinics till the time of visits. Radical treatment (RT) was found to be inadequate and unsupervised. Half of the community leaders were unaware about the availability of diagnostic facility for malaria at these dispensaries and had inadequate information about availability RT.

Mean time interval between onset of fever and BSE was 1.23 days, being more in case of females. Mean number of days between BSE and RT administration was 2.3 days. Adequate RT was given in only one-third of the patients and went majorly unsupervised. No survey activities were carried out by the corporation authorities in areas where Pf positive cases were found. Limited active surveillance was also evident from the response of the community leaders where only half of them said that active surveillance was going on in their area.

It is recommended that, for the program to function efficiently, the vacant posts of LT need to be filled up along with improvement in the basic infrastructure facilities for the laboratories. Adequate logistics and supplies for BSE need to be provided to the malaria dispensaries. Stepping up of active surveillance activities in urban areas along with a system to calculate the monthly blood examination targets is required. The new guidelines for treatment of malaria need to be distributed to all concerned in the corporation dispensaries. Extra efforts are needed in dispensing the correct dose of the medicine to the patients and ensuring supervised treatment.

\section{Acknowledgements}

The authors would like to acknowledge Dr. VS Mazumdar, Professor and Head, Department of Preventive and Social Medicine, Medical College Baroda, for his constant support throughout the study period and all the colleagues of the Department who helped in the data collection process. We sincerely thank the officials from the Municipal Corporation of the district for permitting us to conduct the study. The study was conducted through financial support from Director Office, NVBDCP, and Government of Gujarat. 


\section{References}

1 El-Moamly A (2013) Malaria elimination: needs assessment and priorities for the future. J Infect Dev Ctries 7: 769-780.

2 Shiv L, Sonal GS, Phukan PK (2000) Status of Malaria in India. J Indian Acad Clin Med 5: 19-23.

3 Misra S, Shringarpure K (2015) Perceptions of Field Workers and Community Leaders Regarding Vector Control Activities in Urban Vadodara: Excerpts from the Field. J Infect Dis Ther 3: 246.

4 Kumar A, Valecha N, Jain T, Dash AP (2007) Burden of malaria in India: retrospective and prospective view. Am J Trop Med Hyg 77: 69-78.

5 National Framework for Malaria Elimination in India 2016-2030 (2016) Directorate of National Vector Borne Disease Control Programme (NVBDCP), Directorate General of Health Services (DGHS), Ministry of Health \& Family Welfare, Government of India.

6 Solanki DM, Misra S (2007) Evaluation of operational efficiency of the national anti-malaria program in "High-Risk" rural areas of Vadodara district. Indian J Community Med 32: 249-254.
7 Yasuoka J, Jimba M, Levins R (2014) Application of loop analysis for evaluation of malaria control interventions. Malaria journal 13: 140.

8 Berg H, Velayudhan R, Ebol A, Catbagan BH Jr, Turingan R, et al. (2012) Operational efficiency and sustainability of vector control of malaria and dengue: descriptive case studies from the Philippines. Malaria journal 11: 269.

9 Dean AG, Arner T, Sunki GG, Friedman R, Lantinga M, et al. (2011) Epi Info $^{\mathrm{TM}}$, a database and statistics program for public health professionals, USA.

10 National Vector Borne Disease Control Programme (2016) Directorate General of Health Services, Ministry of Health \& Family Welfare, Urban Malaria Scheme.

11 Kishore J (2005) National Health Programs of India: National Policies \& Legislations related to Health. 6th edn. Century Publications, New Delhi, India.

12 World Health Organization (2014) Global Technical Strategy for Malaria 2016-2025. 